\title{
In Situ Neutron Diffraction Studies of Increasing Tension Strains of Superelastic Nitinol
}

\author{
Alan R. Pelton ${ }^{1} \cdot$ Bjørn Clausen ${ }^{2} \cdot$ Aaron P. Stebner $^{3}$
}

Published online: 4 September 2015

(C) ASM International 2015

\begin{abstract}
A micromechanical study of the effect of varying amounts of tensile strains on the microstructures and subsequent mechanical behaviors of superelastic Nitinol rods is presented. It is found that strains up to $\sim 8-9 \%$ develop microstructures that assist both forward and reverse transformation relative to un-strained material. This superelastic phenomenon is explained to be analogous to two-way shape memory effect in Nitinol actuation materials. These results provide understanding as to why such "pre-strains" may lead to improvements in subsequent superelastic fatigue life. Beyond $9 \%$, a drastic change is observed, as large amounts of martensite (75\% and more) are retained in unloaded samples. Thus, a competition between transformation, plasticity, and reorientation is found to give rise to microstructures that inhibit complete transformation. Furthermore, even though similar inelastic strain magnitudes are observed in loading and unloading plateaus, micromechanical mechanisms differ substantially from samples with less pre-strain. For example, in highly pre-strained samples at least half of the plateau strains are due to martensite reorientation, whereas, in low and moderately pre-strained samples nearly the entirety of the plateau strain is due to transformation. We also find that latent heat of plastic flow is larger than latent heat of transformation.
\end{abstract}

Aaron P. Stebner

astebner@mines.edu

G. Rau, Inc., Fremont, CA 94536, USA

2 Los Alamos National Laboratory, Los Alamos, NM 87545, USA

3 Department of Mechanical Engineering, Colorado School of Mines, Golden, CO 80401, USA
Keywords Micromechanics - Martensitic phase transformation · Plasticity $\cdot$ Shape memory alloy $\cdot$ Fatigue

\section{Introduction}

Many superelastic nickel-titanium (Nitinol) medical devices are inherently pre-strained due to the process of "crimping" an oversized structure into a smaller profile delivery system prior to deployment in the human body, as is the case for pulling self-expanding stents into a catheter $[1,2]$. Although the maximum crimp strains may only occur within small, local volumes of the medical device (for example, at the joints of diamond-shaped stent struts), these same regions typically dictate the maximum strains during in vivo physiological loading. Hence they are the critical regions in devices for fatigue. Thus, understanding the mechanics of these regions is important for predicting long-term durability of implanted medical devices. Of particular interest are the mechanics of "crimping" a device into a catheter, a process that imposes locally large pre-strains in these critical regions, and how post-crimp deformation proceeds once the device is implanted. Patents have been issued and empirical observations have been made that indicate certain pre-straining practices of superelastic nickel-titanium (Nitinol) may lead to substantial improvements in simulated in vivo fatigue performances [3-7]. Nevertheless, it is not universally known whether crimping strains, which are usually 6-14\% equivalent strain in magnitude, provide beneficial or detrimental effects for subsequent in vivo musculoskeletal and cardiac deformation out to $10^{7}-10^{9}$ cycles (i.e., 10-15 year equivalent lifetime). In a recent rotary bend fatigue study of medical-grade Nitinol wire, it was found that fatigue life does not correlate with the amount of pre- 
strain. Instead the data indicate that the optimal pre-strain appears to lie within a narrow deformation region and that compressive bending pre-strains have a negative effect on subsequent rotary bend fatigue life [8].

The effects of pre-strain or "training" has long been recognized in the shape-memory actuator field [9-15]. These references indicate that there are optimal combinations of thermomechanical processing with subsequent prestraining (straining over that of the application) that tends to stabilize the microstructures for either stress or strain cycling. To date, however, characterizations of this phenomenon have been largely phenomenological with little insight into the underlying microstructural changes. For example, patents on this topic indicate that roughly $8 \%$ tensile pre-strain is the "magic" number [6], or in other cases it is loading to between 700 and $1600 \mathrm{MPa}$ during the pre-strain process [7]. The latter patent provides some micromechanical insight that states a combination of defects plus the existence of "one or more" phases (austenite + possibly retained martensite) which leads to improved fatigue performance, and that pre-strain is one way to create such microstructures. Another method is to retain cold work imparted to materials during processing [7]. Medical devices are processed for use using a combination of these two approaches; i.e., thermomechanical processing of the starting material (i.e., wires, tubes, and sheet/ribbon) followed by shape setting into the device form and then crimping into a delivery system [1, p. 213]. Each step in these complex methods results in varying amounts of deformed austenite, deformed martensite, plasticity, and stress relief. As such, a uniform understanding of the mechanisms that affect in vivo fatigue performance is still lacking. Therefore, to enable physicsbased modeling and calculation of proper processing, including cold work, thermal treatments, as well as prestrain processing of complex Nitinol structures, we must first generate data and understanding of the microstructure mechanisms.

In a parallel work, we laid a foundation for such understanding by investigating the monotonic tension (up to $10 \%$ ) and compression (up to $-8 \%$ ) deformations of the same Nitinol rods with in situ neutron diffraction during mechanical loading. We observed a rather abrupt shift in deformation mechanisms around 8.50/-4.75\% tension/compression engineering strain that leads to a relaxation of internal stresses. These stress relaxations were large enough to cause reverse transformation in the static equilibrium responses of the sample microstructures, even as monotonic increased loading was continued [16]. In this work, we turn our attention now to document the specific effects of varying amounts of pre-strain on the unloaded microstructures and subsequent deformation mechanics of the same Nitinol rods in tension. We will use these insights to help explain the seemingly diverse observations of the effects of pre-strain on fatigue performance of Nitinol medical devices.

\section{Methods}

\section{Materials}

The material in this work is the same as in [16], as is the ensuing description of the material. Commercial-scale lowoxygen grade (extra low inclusion, or "ELI") $\mathrm{Ni}_{50.8} \mathrm{Ti}_{49.2}$ (in atomic percent) was manufactured by multi-cycle vacuum-arc remelt processing at ATI Metals, Wah Chang (Albany, Oregon, USA). The chemical composition and inclusion distribution was characterized in the wrought material at a 25.4-mm-diameter bar size in accordance with ASTM F2063 [17]. ELI Nitinol has a significantly lower oxygen $(<60$ mass ppm) and carbon $(<20$ mass ppm) content than the ASTM specification that results in maximum inclusion length of $17 \mu \mathrm{m}$ and area fraction of nonmetallic inclusions of $0.28 \%$. The fully annealed $A_{\mathrm{f}}$ of the bars was $-11^{\circ} \mathrm{C}$.

The ELI Nitinol bars were then cold drawn with successive $35 \%$ cross-sectional area reductions and subsequent stress-relief heat treatments at $510{ }^{\circ} \mathrm{C}$ for $5 \mathrm{~min}$ prior to machining the specimens from $10-\mathrm{mm}$ diameter rods. Cylindrical dogbone tensile specimens with a $20.3-\mathrm{mm}$ radius taper from 8 -mm-diameter threaded grip sections to gage sections of $5.10 \mathrm{~mm}$ diameter $\times 15.25 \mathrm{~mm}$ long were machined on a lathe. The specimens were then stress relieved at $525{ }^{\circ} \mathrm{C}$ for $5 \mathrm{~min}$ to achieve an $A_{\mathrm{f}}$ of approximately $17^{\circ} \mathrm{C}$. The corresponding Differential Scanning Calorimetry (DSC) data have been published elsewhere [16]. The target $A_{\mathrm{f}}$ temperature is approximately $10{ }^{\circ} \mathrm{C}$ below the in situ deformation and diffraction temperature of $\sim 30^{\circ} \mathrm{C}$, allowing for study of stress-induced martensite.

\section{Diffraction Instrument}

The SMARTS instrument at the Manuel Lujan Jr. Neutron Scattering Center of Los Alamos National Laboratories has been reviewed elsewhere [18]. In review, we used a horizontal load frame oriented at $45^{\circ}$ relative to the incident beam of neutrons. Boron-nitride slits masked the beam to a $5 \times 5 \mathrm{~mm}^{2}$ cross-sectional area. The detectors on either side of the specimen recorded data with diffraction vectors within $0^{\circ}-11^{\circ}$ alignments of parallel $\left(\mathbf{Q}_{\mid},-90^{\circ}\right.$ bank $)$ and perpendicular $\left(\mathbf{Q}_{\perp},+90^{\circ}\right.$ bank) to the applied load. Diffraction spectra (effective range of $0.6-3.5 \AA$ ) were recorded simultaneously in each detector. In the ensuing work, we document uniaxial deformation in the direction of 

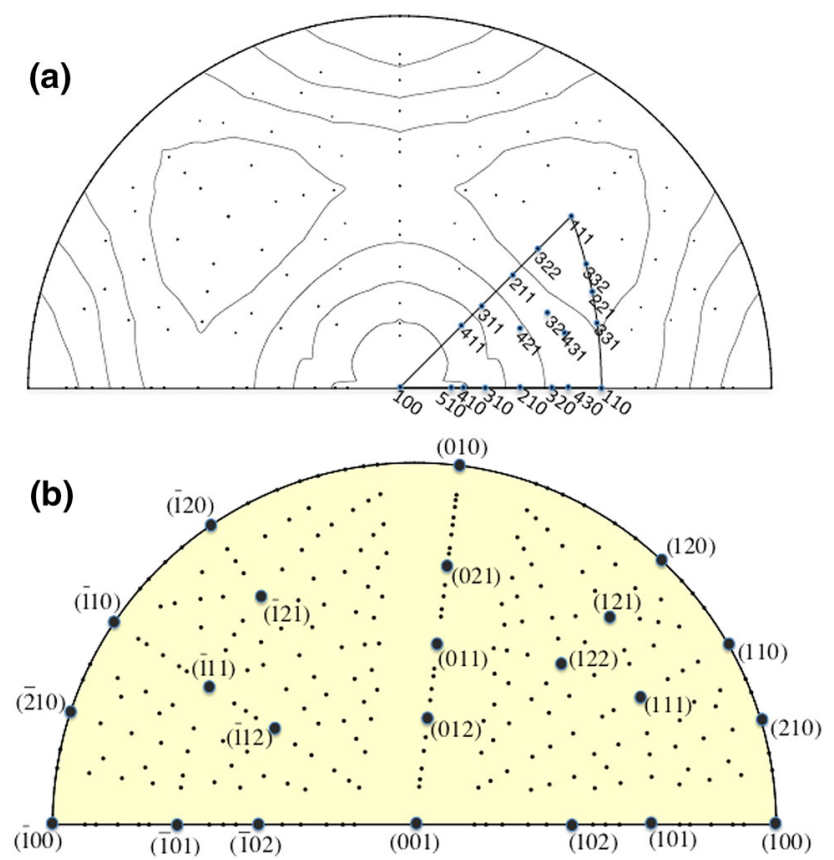

Fig. 1 Inverse pole figures showing the experimental coverage for a B2 (austenite) and b B19' (martensite) measurements. Each dot indicates a peak contained within the diffraction spectra. All measured reflections are labeled for B2 (considering material symmetry), while low-index reflections are labeled for B19'

the applied load, thus we report data from the $\mathbf{Q}_{1},-90^{\circ}$ bank. These spectra contain the reflections indicated with markers on inverse pole figures in Fig. 1, which is reprinted here from [16].

\section{Loadings and Measurements}

Specimens were mechanically loaded at RT $\left(\sim 30^{\circ} \mathrm{C}\right)$ in the beam's path. Macroscopic strain was measured using a mechanical extensometer that spanned the irradiated region. Diffraction patterns were recorded for roughly $40 \mathrm{~min}$ at the points indicated in Table 1. For Sample 3, extensometer slippage occurred while loading to peak load due to surface roughness changes caused by martensitic transformation. We corrected the max load and unloading data using the crosshead displacement versus load relations for Sample 1. Loading of Sample 3 is not shown, as the strain rate was higher than loading Sample 1, thus the strain data could not be corrected in the same manner. The mechanical loading and unloading engineering strain rate was $5 \times 10^{-4} \mathrm{~s}^{-1}$ in all cases, except when the extensometer slipped in loading Sample 3. Mechanical loading paths were carried out in engineering strain control, except the "Initial" and "Unload" data points, which were held in load control at $0 \mathrm{MPa}$. Sample and actuator temperatures were measured with 3 thermocouples-one placed on the actuator piston, one placed on the sample, near the grip, and one placed on the gage of the sample. These thermocouples were tied onto the surfaces of indicated points with thermocouple wire. We report data only for the sample gage thermocouple, as the actuator temperature was not observed to shift by more than $2{ }^{\circ} \mathrm{C}$ relative to the ambient temperature in the hutch, which drifted by several degrees over the days these tests were run, as evident in the drifting baseline temperatures in Figs. 2c and 3c.

\section{Neutron Data Analysis}

Neutron data were binned and fits to 0.6-3.6 ̊ diffraction patterns were refined using the SMARTSware [19] routine SMARTSRunRep, which performs sequences of Rietveld refinements [20], which are used to calculate inverse pole figures (IPFs), by calling sequences of the GSAS programs EXPEDT, RAWPLOT, POWPREF, and GENLES [19, 21, 22]. We used the crystal structures reported by Kudoh et al. [23] for initializing each monoclinic and cubic phase in the refinements. Atomic positions and thermal motions were held fixed during the refinements. Austenite (B2) diffraction patterns were refined using space group $\mathrm{Pm} \overline{3} \mathrm{~m}$ and a tenth-order spherical harmonics model for texture; monoclinic (B19') diffraction patterns were refined using space group P $11122_{1 / m}$, and an eighth-order spherical harmonics texture model. Lower order texture models were used in cases where phase fractions of austenite or martensite were less than $5 \%$. Our threshold for being able to meaningfully refine the martensite phase was around $2.5 \%$ weight fraction. For data points with less than $2.5 \%$ martensite, we use a $0 \%$ mean weight fraction with an errorbar of $3 \%$. Lattice strains of B2 reflections were calculated using the SMARTSware routine SMARTSSPF to fit individual reflections using the GSAS time of flight profile function 3 (TOF-3). They were calculated relative to the initial, unloaded state of the material. Thus the values reported in this work represent changes due to mechanical loading, but do not incorporate strains inherent to the processing.

\section{Results}

Figure 2 shows mechanical, phase fraction, and temperature responses of the gage of Sample 1, while these same responses are shown for Samples 3, 7, and 8 in Fig. 3. The stress relaxations at load and strain recoveries upon unload of Sample 1 occur during the $\sim 40$ min scan times, mostly in the first minute, as for the stress relaxations at load and rises during unload for Samples 3, 7, and 8. Sample 7 is the same tension sample reported upon in the parallel work [16]. However, we report the data here in a slightly different manner than we did previously to facilitate 
Table 1 Load paths and scanning regimes for the data presented in this study

\begin{tabular}{|c|c|c|c|}
\hline ID & Test description & \# scans & Scan points (\% engineering strain) \\
\hline 1 & Load to increased maximum strain amplitude each cycle & 15 & $\begin{array}{l}\text { Initial, 0.50, Unload, 2.00, Unload, 4.00, Unload, 6.00, } \\
\text { Unload, 8.00, Unload, 10.00, Unload, 12.00, Unload }\end{array}$ \\
\hline 3 & Load to $13.80 \%$ strain, unload, reload, unload & 16 & $\begin{array}{l}\text { Initial, } 1.00,13.80,13.30,12.30,10.30,8.30,7.30,6.30 \text {, } \\
\quad 4.80,2.80,3.30,4.80,6.30,7.30 \text {, Unload }\end{array}$ \\
\hline 7 & Load to $11.00 \%$ strain, unload & 40 & $\begin{array}{l}\text { Initial to } 1.00 \text { in } 0.20 \text { intervals, } 1.25,1.50-11.00 \text { in } 0.50 \\
\text { intervals, } 10.90,10.70,10.50-5.50 \text { in }-0.50 \text { intervals }\end{array}$ \\
\hline 8 & $\begin{array}{l}\text { Load to } 9.00 \% \text { strain, unload, reverse load in } \\
\text { compression }\end{array}$ & 13 & $\begin{array}{l}\text { Initial, } 9.00,5.50,5.00,3.00,1.00,0.50,0.00,-0.50 \\
\quad-1.00,-2.00,-3.00,-4.00\end{array}$ \\
\hline
\end{tabular}

All mechanical loads were applied and removed in engineering strain control at a strain rate of $5 \times 10^{-4}$. At each scan point indicated by an engineering strain value, the samples were held in strain control for $\sim 40 \mathrm{~min}$ while diffraction measurements were made

comparison to the additional samples we study in this work. Figure 4 shows the inverse pole figure (IPF) evolution in the load direction for Sample 1, while Fig. 5 represents the analogous information for Samples 8 and 3, and Fig. 6 for Sample 7. As reported in the parallel paper [16], the initial IPFs (Figs. 4a, 5a, o, 6a) are consistent with $\{111\}_{\mathrm{A}}$ fiber symmetry texture with a stronger intensity of $\{111\}_{\mathrm{A}}$ grains oriented along the fiber than in transverse directions (grains with their $\{110\}_{\mathrm{A}}$ axes aligned with the load direction), which we have confirmed for ELI Nitinol rods in separate measurements made at the advanced photon source (APS). These IPFs indicate roughly the same initial strength of texture. We reserve further interpretation of these figures for the Discussion. Figure 7 depicts the orientation-specific strains $\left(\varepsilon_{h k l}\right)$ in the load direction for high-order B2 reflections from the first five unloaded patterns from Sample 1. The corresponding tensile residual stress calculations made using the B2 elastic constants of Mercier et al. [24] according to the relationship between single crystal elastic constants and $(h k l)$-specific moduli [25]. Since the lattice strains were measured relative to the initial state of the prepared samples (See "Neutron Data Analysis" section), these calculated stresses represent changes to the residual stress states of the samples, but do not reflect the initial residual stress of the material due to processing. Futhermore, it was not possible to accurately fit the B2 lattice strains in isolation for the latter unload events due to two-phase (austenite and martensite) mixtures with commensurate peak overlap that prohibited accurate peak position determination of the $\mathrm{B} 2$ peaks in isolation. Internal stresses of the same reflections measured of Sample 8 near zero load, as well as interpolated zero load values are given in Table 2. Because this sample was unloaded in strain control, and for many strains, the sample passed through zero stress (Fig. 3), the interpretation of the data is not the same as for Sample 1 (Fig. 7). There were many strains during the unload process at which the stress was $0 \mathrm{MPa}$, thus plotting the data directly on Fig. 7 was not straightforward. Hence, to create Table 2, we assessed the residual stress from the lattice strains measured just before and after crossing from tension to compression for the last time during the unloading process, and then interpolated the values at zero stress. Note that $(100)_{\mathrm{A}}$ statistics for Sample 1 were not sufficient to report in Fig. 7, but for Sample 8, slightly longer measurements were made, and better statistics attained allowing the incorporation of $(100)_{\mathrm{A}}$ in Table 2.

\section{Discussion}

\section{Effects of Tensile Pre-strain Less than $8 \%$}

Several trends emerge in the data from the $0.50,2.00,4.00$, and $6.00 \%$ pre-strain events of Sample 1 that are worth noting. First, in spite of these deformations being well within the plateau regime (Fig. 2a), unrecovered strain does accumulate with each cycle, albeit very small amounts $(0.01,0.04,0.08$, and $0.13 \%$ engineering strain, respectively, Fig. 7). Second, these cycling events result in a buildup of $50 \mathrm{MPa}$ maximum tensile stress in $\{110\}_{\mathrm{A}}$ grains. Third, concomitantly, the transformation initiation stress drops with each cycle (Fig. 2a), consistent with earlier studies (e.g., [26]). Fourth, the residual B2 change in poles aligned with the load (Fig. 4k) is nearly identical to the change caused by the first grains to transform (Fig. 4b).

While we do not directly observe dislocation structures in the diffraction patterns, all of these results are consistent with an active mechanism of transformation-induced plasticity in shape memory alloys (SMAs). Research on transformation-induced plasticity spans several decades; these studies show that dislocation structures form and get pushed through the material along interfaces, thus remnant dislocation structures often resemble the austenite/martensite or martensite/martensite interfaces themselves [12, 27-32]. These dislocation structures provide "vestigial marks" that act as "microscopic parking lots" (to quote the late Prof. Jeff Perkins [32]) and both reduce 
Fig. 2 The responses of Sample 1, which was cycled with increasing strain amplitude and unloaded after each cycle are shown: a the engineering stress-strain response, $\mathbf{b}$ the phase fraction versus strain response, and $\mathbf{c}$ the temperature versus strain response. Note that in $\mathbf{b}$ lines connect markers to help guide the eye through the cycling sequence; phase fraction measurements were only made at peak load and unload for each cycle, and based on the results shown in Fig. 3, the evolution of phase fraction is not expected to be linear for each cycle

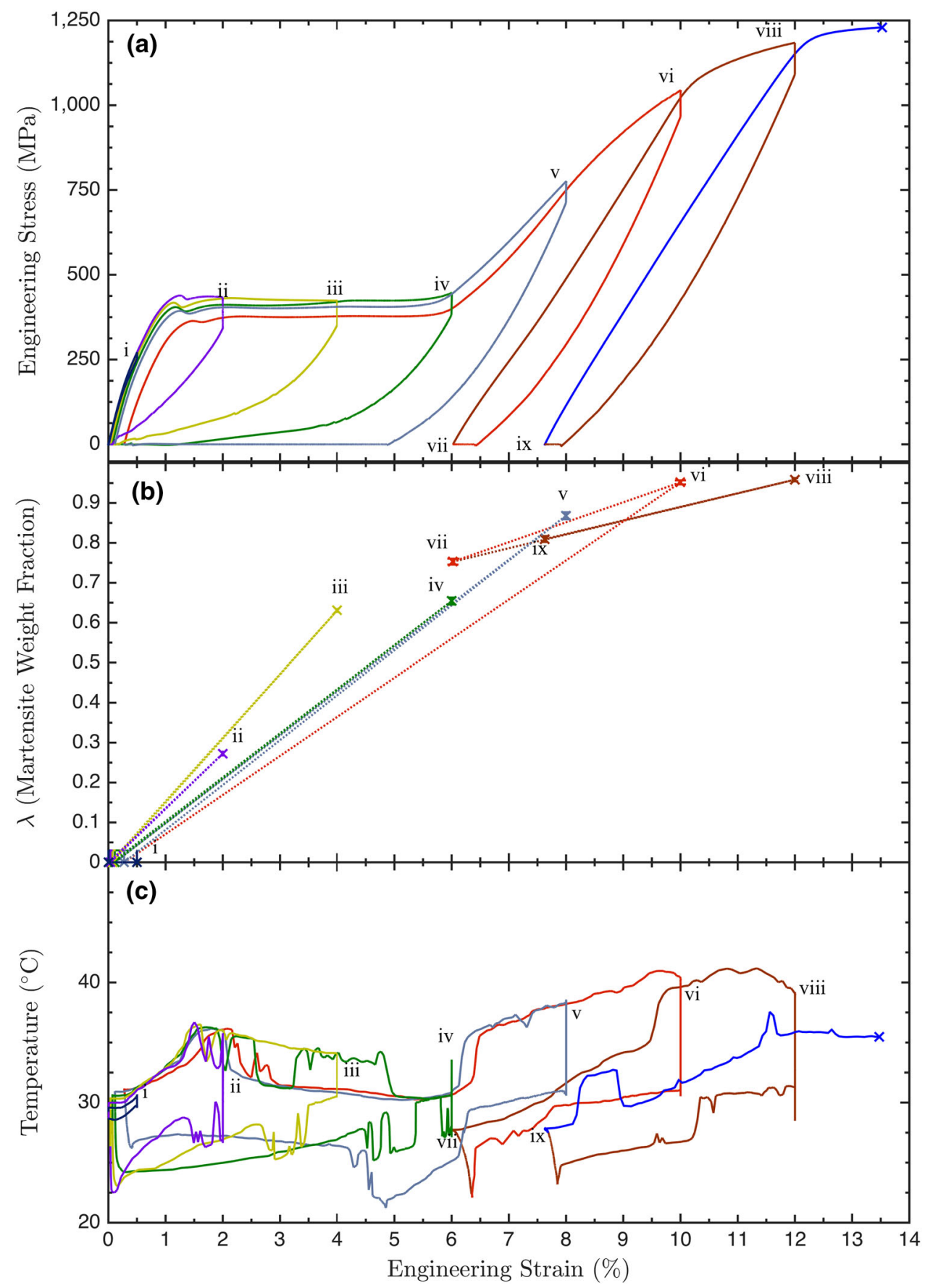

the driving force needed for and guide martensite formation in subsequent cycles. The dislocation structures are necessary because the austenite and martensite lattices in Nitinol cannot form theoretically ideal coherent interfaces with each other [33].

\section{Effects of Tensile Pre-strain Between 8 and $10 \%$}

In comparing the IPFs in Fig. 4 with those in Fig. 6, the nature of the IPFs does not change due to cycling versus monotonic loading to $8 \%$ strain. This is an important observation in that the width of the superelastic plateau strain is nominally $6 \%$; therefore, loading between 6 and $8 \%$ strain invokes alternative accommodation mechanisms, as discussed in detail in [3]. There are some differences in intensities, as the martensite texture is stronger in the cycled sample and the austenite texture is weaker. However, at $8 \%$ strain, Sample 1 has $~ 90 \%$ martensite while Sample 7 $80 \%$ (Figs. 2, 3), so these differences are consistent, as are subtle differences at 2 and $6 \%$ strain, analogously. 
Fig. 3 The responses of Samples 3, 7, and 8, which were loaded to different maximum strains, unloaded in strain control, and then reverse loaded into compression: a the engineering stress-strain response, $\mathbf{b}$ the phase fraction versus strain response, and $\mathbf{c}$ the temperature versus strain response. Note that as discussed in "Methods" section, the extensometer slipped while loading Sample 3, thus only the unload and reverse load data are shown for this sample

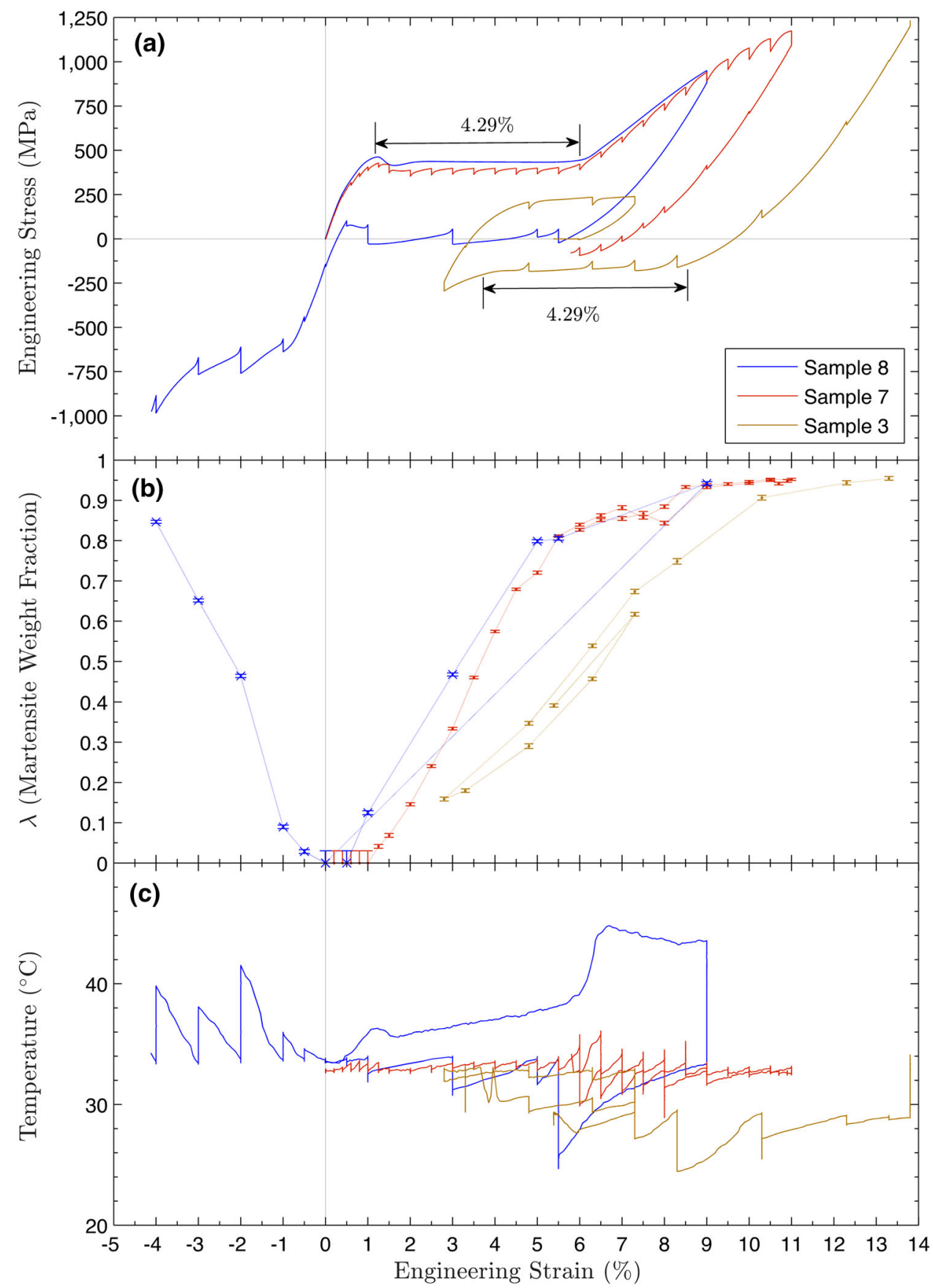

A very interesting phenomenon is observed of Sample 1 after $8 \%$ pre-strain. Upon unloading at an engineering strain rate of $5 \times 10^{-4}$, there is $5 \%$ unrecovered strain, $4.7 \%$ of which recovers in the first minute of the hold at $0 \mathrm{MPa}$ to collect neutron data (Fig. 2a). This behavior indicates strong rate dependence from the material, even at rates that are normally thought to be quasi-static (i.e., low enough rates that dynamic effect are not expected to dominate). Furthermore, it seems that reverse transformation and retained martensite are near a static equilibrium, though reverse transformation is still slightly favored, as nearly all of the material transforms back to austenite (Fig. 2b). The maximum residual tensile stress doubles in $\{110\}_{\mathrm{A}}$ grains to $\sim 100 \mathrm{MPa}$ relative to unloading after $6 \%$ pre-strain, and the disparity between the strongest and weakest grains also increases. Interestingly, the ratio of maximum to minimum residual stress is approximately constant at 2 amongst the orientations we measured (Fig. 7).

In pre-straining Sample 8-9\% strain, an analogous quasi-static, near-equilibrium phenomenon of reverse 


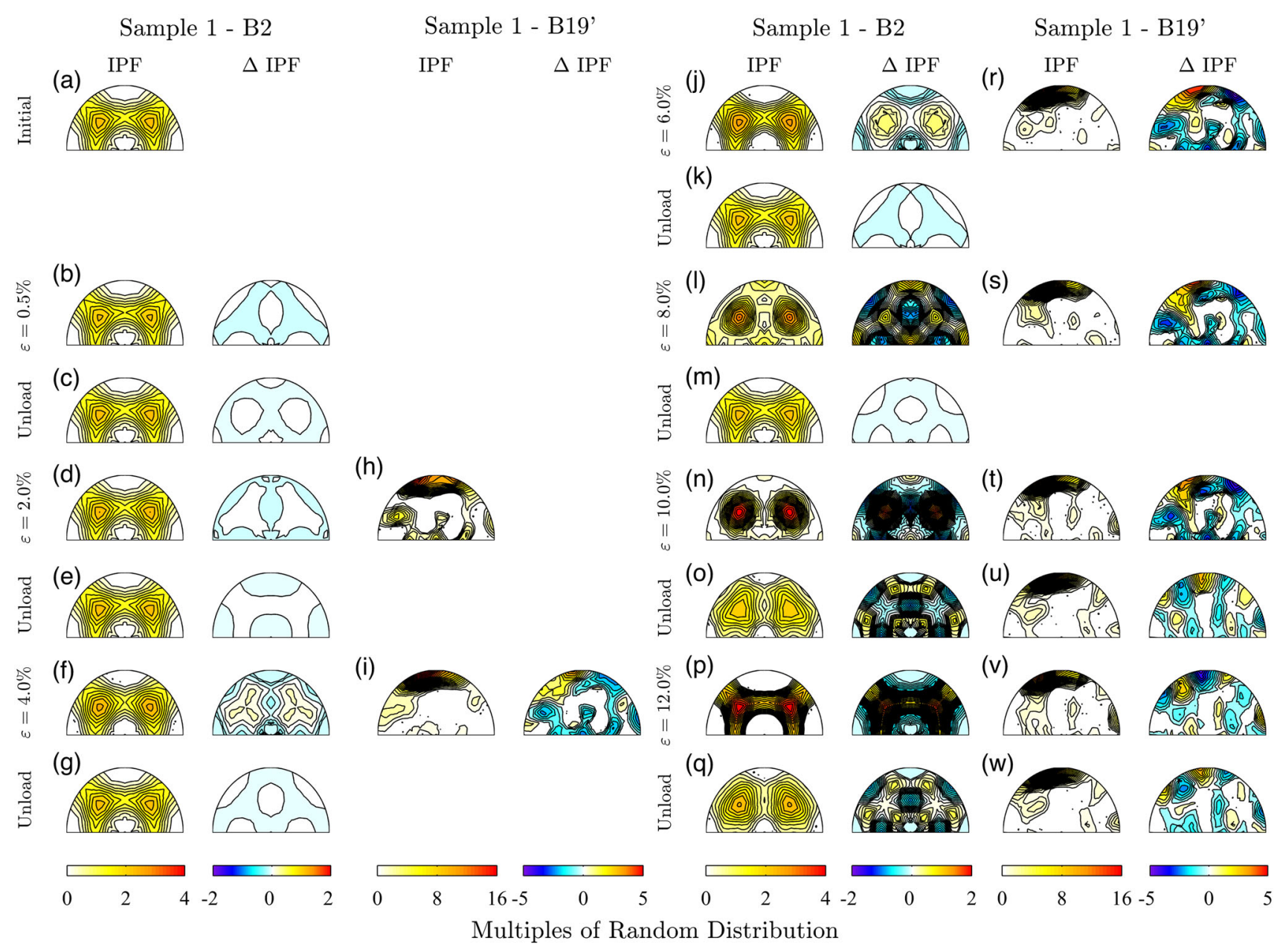

Fig. 4 Selected inverse pole figures (IPFs) in the load direction measured of Sample 1. Differences between each B2 IPF and a the initial B2 IPF are shown for the austenite phase. Differences between $\mathbf{i}, \mathbf{r}-\mathbf{t}$ B19' IPFs up to $10 \%$ strain and $\mathbf{h}$ the first measured B19' IPF

transformation versus retained martensite is observed. However, under these strain conditions the sample is unloaded in strain control, and must be driven into compression to decrease strain, but then the macroscopic stress becomes tensile during the neutron diffraction measurements (Fig. 3a). Thus the $5 \times 10^{-4}$ unloading plateau is compressive, but the static plateau is tensile in nature. Reverse transformation (practically) completes at an unloaded strain of $0.5 \%$, and further reverse loading drives the sample into elastic compression loading, and then forward transformation in compression (Fig. 3b). Residual stresses in this material are slightly greater than after $8 \%$ pre-strain, with maximum values of $\sim 125 \mathrm{MPa}$ (Table 2).

Finally, the equilibrium point between reverse transformation and retained martensite is bypassed in prestraining Sample $1-10 \%$, as $\sim 75 \%$ phase fraction is retained upon unloading (Fig. 2). While we could not measure residual stresses in the material due to the high are shown, and then differences between each previous figure are taken for $\mathbf{u}, \mathbf{v}$ for the martensite phase. Note that the scalebars are different for the B19' phase relative to the B2 phase as the distributions are more sharply focused

phase fraction of 2-phase mixture, we know from the trends of Samples 1 and 8 that more than $125 \mathrm{MPa}$ bulk residual tensile stress is required to stabilize martensite. Note that the macroscopic load needed to drive bulk transformation in a subsequent mechanical cycle of the material is lowered from 440 to $360 \mathrm{MPa}$ as a result of $8 \%$ pre-strain (Fig. 2a). This finding, together with the nearly full recovery of strain while holding at 0 load, indicate that in this condition the resistance of the material to passing transformation fronts is small. In fact, the microstructure appears to be assisting transformation at a rate that is much slower than the bulk deformation strain rate of $5 \times 10^{-4}$, and also much slower than would be expected of a simple slip or twinning-related Bauschinger effect, such as those previously observed in monoclinic NiTi [34] as well as B2 and mixtures of $\mathrm{B} 2+\mathrm{B} 19^{\prime} \mathrm{NiTi}$ [35] at analogous unloading rates. It is likely this addition of both forward and reverse transformation assistance from the 


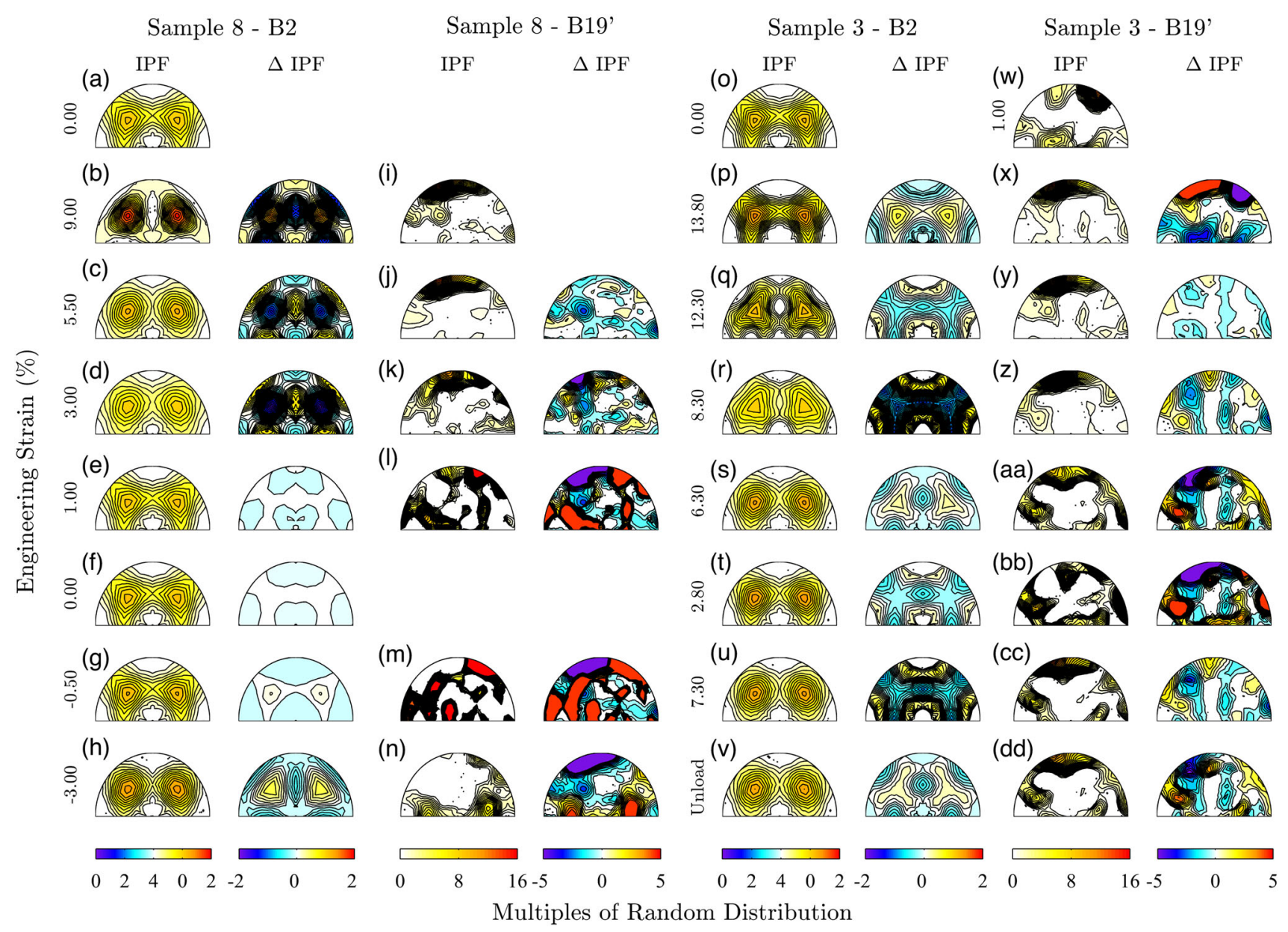

Fig. 5 Selected inverse pole figures (IPFs) in the load direction measured of Samples 8 and 3. For the austenite phase, differences between $\mathbf{b}, \mathbf{e}-\mathbf{h}$ and $\mathbf{p}, \mathbf{s}-\mathbf{v}$ are shown relative to initial B2 IPFs (a, o), while the differences for between $\mathbf{c}, \mathbf{d}$ and $\mathbf{q}, \mathbf{r}$ are shown relative to max load IPFs (b) and (p) for Samples 8 and 3, respectively. For

microstructure "parking lots" that gives rise to improved fatigue life. That is, the creation of biased residual stress together with defect structures can assist in the forward and reverse transformation, whether through thermal or mechanical events. This concept is not foreign to SMAsit is the superelastic analog of two-way shape memory effect and enhanced thermal actuation after a few temperature-high stress cycles. Indeed, the concept of cyclic "training" and two-way memory was incorporated in the early (1970's) heat engines to stabilize the cyclic response of Nitinol wires [36]. More recently, in fully annealed thermal martensite, optimal two-way shape memory effect was imparted with a tensile pre-strain greater than $6 \%$, and for sure between 10 and $14 \%$, with degradation of behavior thereafter (pre-strains between 6 and $10 \%$ were not studied) [37]. Despite the differences in manner of actuation (thermal versus mechanical) as well as composition and thermomechanical processing, the current results martensite, all difference IPFs for Sample $8 \mathbf{j}-\mathbf{n}$ ) are taken with respect to peak load (i), while for Sample 3, $\mathbf{x}$ is taken with respect to (w), and all remaining difference IPFs $\mathbf{y}-\mathbf{d d}$ are taken with respect to (x). Note that the scale bars are different for the B19' phase relative to the B2 phase as the distributions are more sharply focused

and those from, for example, Benafan et al. [37], are comparable findings. They concur with patents [3-7] and personal communications with Launey et al. that shows fatigue enhancement with tensile pre-strain in Nitinol wires [8]. Hence, we propose that pre-strain of actuators to this same extent, in a microstructural sense, would increase their fatigue performance. This is supported by the fact that pre-strained superelastic material exhibits a strong twoway effect if cryogenically cooled below the martensite finish temperature and then re-heated above $\mathrm{A}_{\mathrm{f}}$.

Martensite is significantly retained after strains beyond $9 \%$ pre-strain, as the material goes from (nearly) full recovery of martensite to a $75 \%$ stress-stabilized phase fraction of martensite upon unload. Although we could not quantify the exact residual tensile stress needed for this phenomenon, it is likely greater than $150 \mathrm{MPa}$ in the strongest austenite grains based on the trend in Fig. 7 and Table 2. This is probably the shift that leads to fatigue 


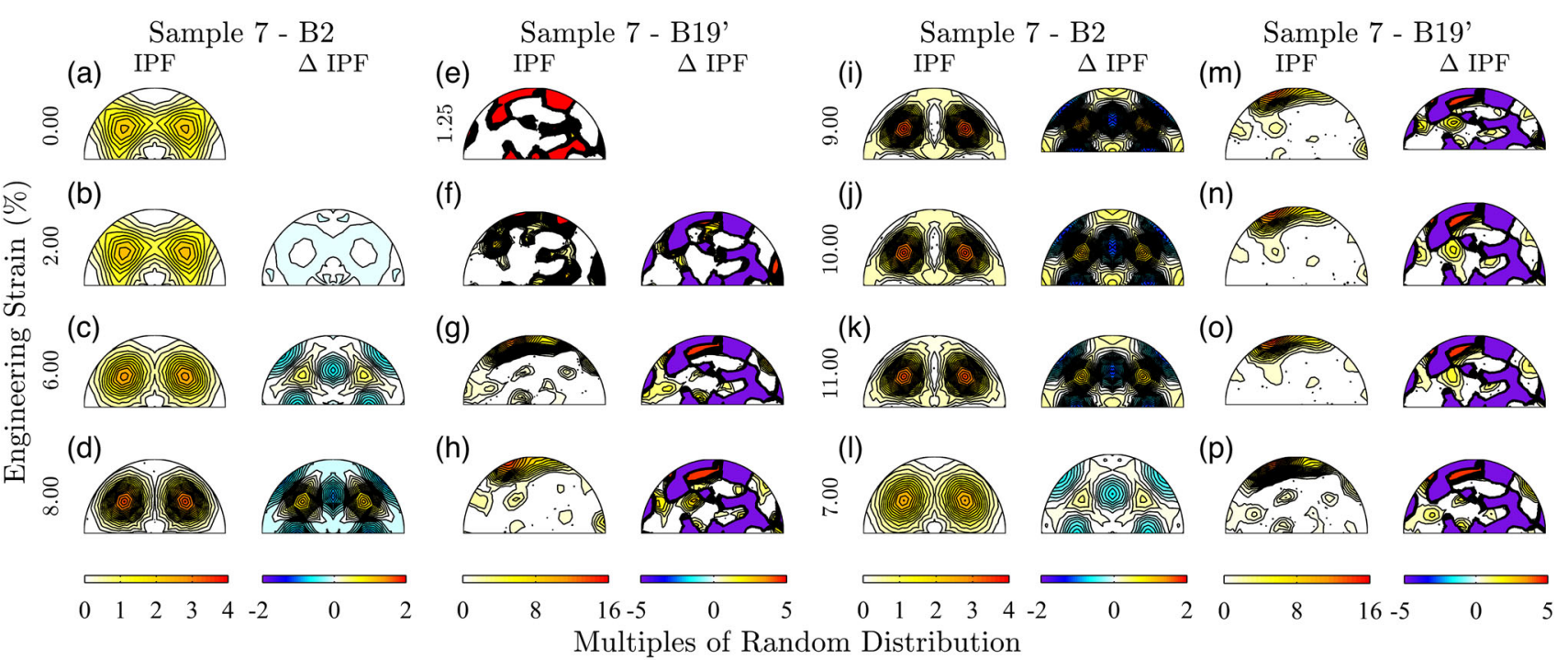

Fig. 6 Selected inverse pole figures (IPFs) in the load direction measured of Sample 7, as well as differences between each IPF and a the initial B2 IPF for the austenite phase and $\mathbf{e}$ the first measured
B19' IPF for the martensite phase. Note that the scale bars are different for the B19' phase relative to the B2 phase as the distributions are more sharply focused
Fig. 7 a Orientation-specific strain changes $\left(\varepsilon_{-}(h k l)\right)$ of highorder $\mathrm{B} 2$ reflections are shown for the first five unload events of Sample 1. Fitting error bars are smaller than the marker used for each data point. b The B2 elastic constants reported by Mercier et al. [24] and $\varepsilon_{-}(h k l)$ were used to calculate changes of the residual stress component in the load direction for each of the reflections

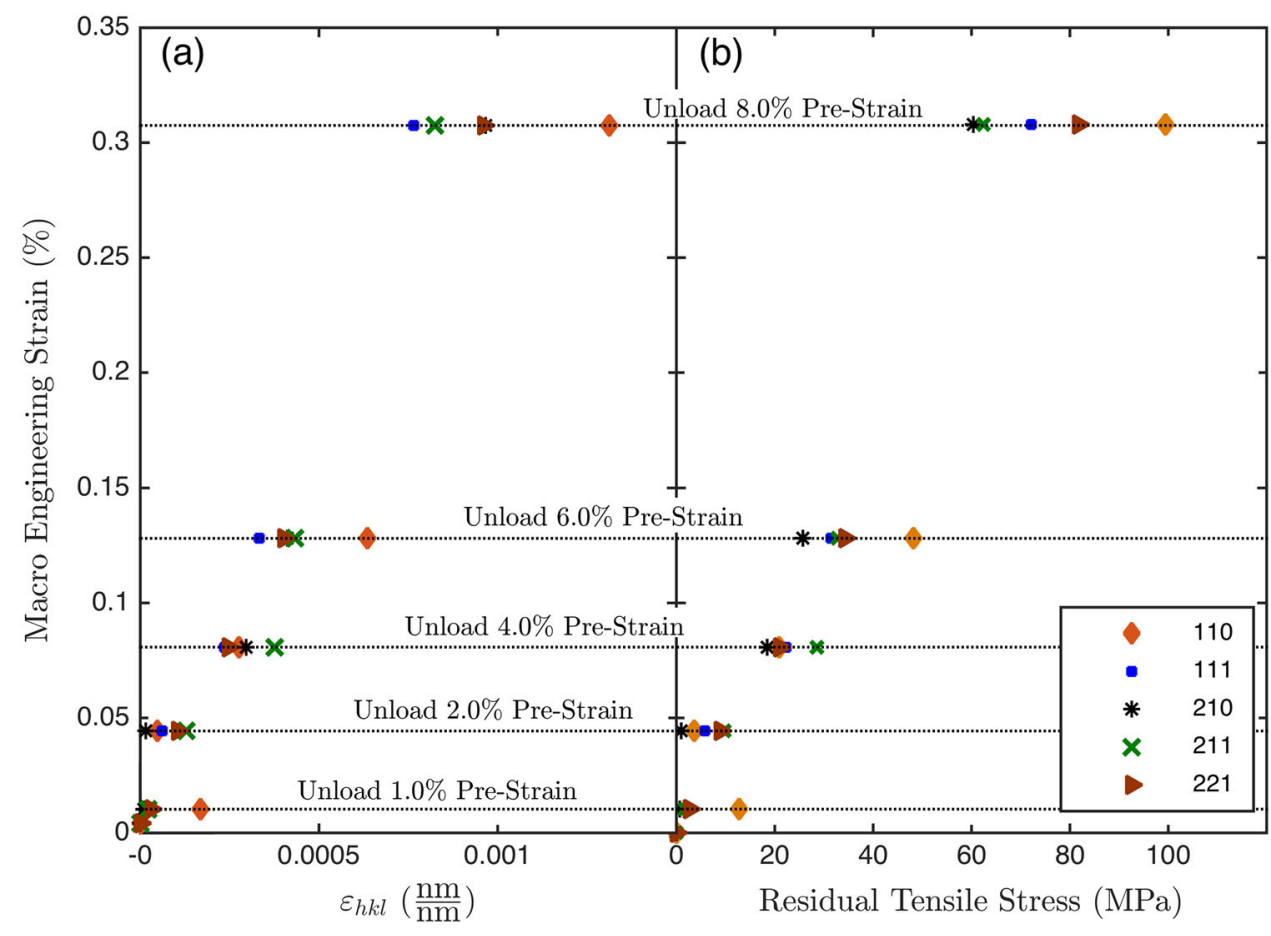

degradation, together with possibly additional surface damage in the case of bending that act to promote crack propagation, as observed by Gupta et al. [8]. This mechanistic shift serves to set up strong competition between martensite reorientation, phase transformation, and plasticity. However, this competition is not completely obvious in the IPF difference plots of Fig. 4o, u, which were calculated with respect to Fig. 4a, t, respectively. Visually, it is difficult to determine the exact cause of the pronounced increases in $\{211\}_{A}$ orientations and concomitant decreases in $\{110\}_{\mathrm{A}}$ orientations in the unloaded B2 IPF relative to the initial IPF. It could be due to (1) deformation twinning or (2) merely due to more of the martensite formed of $\{110\}_{\text {A }}$ grains being retained, while more of the martensite of $\{211\}_{\text {A }}$ grains transformed back during unloading. However, these orientations are not related by $\{112\}_{\mathrm{A}}$, 
Table 2 Internal stresses measured of different B2 reflections after $9.00 \%$ tensile pre-strain, plus approximated residual stresses, all values in $\mathrm{MPa}$

\begin{tabular}{lllllll}
\hline Macro load & $(100)_{\mathrm{A}}$ & $(110)_{\mathrm{A}}$ & $(111)_{\mathrm{A}}$ & $(210)_{\mathrm{A}}$ & $(211)_{\mathrm{A}}$ & $(221)_{\mathrm{A}}$ \\
\hline 103.50 & 94.0 & 246 & 278 & 157 & 187 & 237 \\
-141.85 & -81.9 & -65.3 & -80.7 & -94.0 & -88.7 & -71.1 \\
$0^{\mathrm{a}}$ & 19.8 & 115 & 126 & 51.4 & 70.7 & 107
\end{tabular}

${ }^{\text {a }}$ Residual stress values at $0 \mathrm{MPa}$ load were linearly interpolated between the measurements at 103.50 and $-141.85 \mathrm{MPa}$ for purposes of comparison to Sample 1, Fig. 7. This calculation is a reasonable estimate for the bulk material measurements, as measurable differences in transformation or reorientation were not observed between these two data points and the macroscopic response is linear

$\{113\}_{\mathrm{A}}$, or $\{114\}_{\mathrm{A}}$ compound twinning, the most favorable and previously observed B2 twin systems for Nitinol [34, 38-40]. Therefore, these observations are probably due to the transformation mechanism. The same ambiguity exists in distinguishing martensite reorientation versus martensite that transforms, only here there are multiple increasing and decreasing populations of orientations that are aligned with the load. As such, it is difficult to uniquely correlate them with each other. There is probably some martensite reorientation, but we cannot say for sure from these figures, so we proceed to examine this competition in more detail in conditions of pre-strains greater than $10 \%$, where it becomes very evident.

\section{Effects of Tensile Pre-strain Greater than $10 \%$}

The retained martensite phase fraction upon unload increases to $\sim 85-90 \%$, respectively. (Figs. 2, 3) by loading to $11 \%$ pre-strain (Sample 7), $12 \%$ pre-strain (Sample 1), and to $13.8 \%$ pre-strain (Sample 3). Transformation reorientation competition is evident in the IPFs of the compressive and tensile reloading of Sample 3 (Fig. 3a)-see Fig. 5dd. At peak load, the martensite exhibits a characteristic IPF of highly deformed martensite, as we previously documented in deforming thermal martensite [34] as well as this superelastic material [16]. However, even before the material unloads from the tensile strain, a compressive component begins to appear in the IPF near $(\overline{2} 10)_{\mathrm{M}}$. This "compression" component together with a component near $(102)_{M}$ increases during compressive loading to form martensite of a load direction IPF. This IPF is characteristic of compression-loaded martensite at $2.80 \%$ strain $[16,34]$ past the inflection point for the compressive loading plateau. The material has $20 \%$ retained martensite at this point. The width of this plateau is the same as the forward transformation plateau (Fig. 3a), yet $85 \%$ of the material transformed during forward loading, but only $50 \%$ of the material transformed during reverse loading. The point where the forward transformation reaches $50 \%$ martensite is the mid-point of the forward loading plateau. Clearly in the reverse plateau for $13.8 \%$ pre-strain (Sample 3), at least half of the strain comes from martensite reorientation, and not transformation. Thus, the microstructure mechanism that assisted forward and reverse transformation after $8 \%$ strain is now frustrated, likely because of events such as

(1) martensite twinning out of transformation twins configurations (those that can be recovered through phase transformation) and into deformation twins (twins that are not recovered, and then remain twins in the austenite structure), and/or

(2) bulk plastic flow reconfigured the microstructure "parking lots" into topologies that inhibit, rather than facilitate transformation kinematics.

One final observation unrelated to pre-strain, but worth noting, is that the latent heat release of these materials is much greater beyond the transformation plateaus in forward loading (Figs. 2, 3). This finding shows that plastic flow generates much more heat than transformation, since plasticity is more active than transformation in the regions of greater heat release, as we showed in a parallel work [16].

\section{Conclusions}

This study provides micromechanical understanding as to why certain amounts of pre-strain benefit fatigue life of superelastic Nitinol devices while others degrade it. Some deformation serves to impart defect structures that assist both forward and reverse transformation. However, too much deformation leads to higher order deformation mechanisms that impart microstructures that inhibit subsequent transformations. In these tensile experiments, we observed "compression points" where these higher order mechanisms led to compression-favored textures upon unload. The data also suggest analogous treatments will benefit Nitinol actuators. Furthermore, this precise microstructural account of strain accommodation mechanisms of superelastic Nitinol, including plasticity and transformational/reorientation behavior, may explain the enhancement of fatigue behavior with mean strains between $\sim 1.5-6 \%$ [41]. Together with our parallel report of the large tension-compression asymmetry in the critical mechanism transition points [16], these new data also affirm previous speculation of why bending pre-strains of $8 \%$ or more degrade fatigue life [8] while analogous tensile strains improve it $[3,6]$. The defect structures imparted by tensile pre-strains evoke tensile residual stresses, which shifts the unloaded state of the material 
more toward tension-compression symmetry in subsequent cycles.

Our findings suggest that a two-way shape memory optimization experiment matrix may be a assist understanding optimal pre-strain amounts without the need for in situ diffraction experimentation or fatigue testing to billions of cycles. In more complicated structures, however, beyond wires and rods in uniaxial loading, the answer will likely require assistance of robust three-dimensional modeling that is able to predict the shift from transformationassisting defect structures to retained martensite and other transformation-inhibiting phenomena. Tensile pre-strains may not be the answer. Beneficial cold-work boundary conditions will be dictated by material asymmetry and processing anisotropy, together with the stress state of the most critical elements of the structure of the Nitinol device.

Beyond this study in isolation, the data in this work provide a benchmark for micromechanical and macroscopic models alike that aim to simulate transformationplasticity coupling in SMAs and said transition points. The important addition of surface temperature profiles of the samples during deformation are reported, so modelers need not make assumptions of isothermal or adiabatic conditions. Taken together, these data are used to verify a new macroscopic framework developed for use as an engineering tool in another work [42]. Furthermore, it is expected that these findings will help explain observations in Nitinol material with different compositions and thermomechanical histories used for medical devices.

Acknowledgments We thank Nitinol Devices and Components (NDC) for donating the samples for this research; specifically, Max Launey and Lot Vien for assistance with fabrication of the materials. We also thank Thomas Sisneros of Los Alamos National Laboratory for assistance with the diffraction experiments. This work has benefitted from the use of SMARTS at the Lujan Neutron Scattering Center, which was funded by DOE Office of Basic Energy Sciences. Los Alamos National Laboratory is operated by Los Alamos National Security LLC under DOE Contract DE-AC52-06NA25396.

\section{References}

1. Duerig TW, Tolomeo DE, Wholey M (2000) An overview of superelastic stent design. Minim Invasive Ther Allied Technol 9(3-4):235-246

2. Stoeckel D, Pelton A, Duerig T (2004) Self-expanding nitinol stents: material and design considerations. Eur Radiol 14(2): 292-301

3. Dooley BA, Lasley, CC, Mitchell MR, Steele RR, Tittelbaugh EM (2012) Method of making shape memory alloy articles with improved fatigue performance, United States, US8177927 B2

4. Dooley B, Lasley C, Mitchell M, Steele R, Tittelbaugh E (2003) Shape memory alloy articles with improved fatigue performance and methods therefore, United States, US20040216814 A1

5. Dooley BA, Lasley CC, Mitchell MR, Steele RR, Tittelbaugh EM (2010) Controlled prestraining of nickel titanium (nitinol) material; implantable medical devices, United States, US7789979 B2
6. Dooley BA, Lasley CC, Mitchell MR, Steele RR, Tittelbaugh EM (2014) Shape memory alloy articles with improved fatigue performance and methods therefore, United States, US20140207228 A1

7. Schaffer JE (2013) Method for imparting improved fatigue strength to wire made of shape memory alloys, and medical devices made from such wire, United States, US8414714 B2

8. Gupta S, Pelton AR, Weaver JD, Gong X-Y, Nagaraja S (2015) High compressive pre-strains reduce the bending fatigue life of nitinol wire. J Mech Behav Biomed Mater 44:96-108

9. Eggeler G, Hornbogen E, Yawny A, Heckmann A, Wagner M (2004) Structural and functional fatigue of NiTi shape memory alloys. Mater Sci Eng A 378(1-2):24-33

10. Eucken S, Duerig TW (1989) The effects of pseudoelastic prestraining on the tensile behavior and 2-way shape memory effect in aged NiTi. Acta Metall 37(8):2245-2252

11. Miyazaki S, Imai T, Igo Y, Otsuka K (1986) Effect of cyclic deformation on the pseudoelasticity characteristics of Ti-Ni alloys. Metall Trans A 17(1):115-120

12. Perkins J (1973) Lattice transformations related to unique mechanical effects. Metall Trans 4(12):2709-2721

13. Pelton AR, Simpson J, Stoeckel D (1992) Towards the optimization of shape memory actuators: the effects of pre-strained wire. In: Actuator 92, VDI/VDE, Technologiezentrum Informationstechnik GmbH, Hamburg

14. Frenzel J, Burow JA, Payton EJ, Rezanka S, Eggeler G (2011) Improvement of $\mathrm{NiTi}$ shape memory actuator performance through ultra-fine grained and nanocrystalline microstructures. Adv Eng Mater 13(4):256-268

15. Stebner A, Padula S, Noebe R, Lerch B, Quinn D (2009) Development, characterization, and design considerations of Ni19.5Ti50.5Pd25Pt5 high-temperature shape memory alloy helical actuators. J Intell Mater Syst Struct 20(17):2107-2126

16. Stebner AP, Paranjape HM, Clausen B, Brinson LC, Pelton AR (2015) In-situ neutron diffraction studies of the micromechanics of large monotonic deformations of superelastic nitinol. Shape Mem Superelast 1:1-16

17. ASTM-F2063-12 (2010) Standard specification for wrought nickel-titanium shape memory alloys for medical devices and surgical implants, F2063-12

18. Bourke MAM, Dunand DC, Ustundag E (1995) SMARTS-a spectrometer of strain measurement in engineering materials. Appl Phys A 74:s1707

19. Clausen B (1998) SMARTSware Manual, Los Alamos National Laboratory

20. Rietveld HM (1966) A method for including line profiles of neutron powder diffraction peaks in determination of crystal structures. Acta Crystallogr 21:A228

21. Larson AC, VonDreele RB (1986) GSAS, Los Alamos National Laboratory

22. VonDreele RB (1997) Quantitative texture analysis by Rietveld refinement. J Appl Crystallogr 30:517-525

23. Kudoh Y, Tokonami M, Miyazaki S, Otsuka K (1985) Crystalstructure of the martensite in Ti-49.2 at-percent-Ni alloy analyzed by the single-crystal X-ray-diffraction method. Acta Metall 33(11):2049-2056

24. Mercier O, Melton K, Gremaud G, Hägi J (1980) Single-crystal elastic constants of the equiatomic NiTi alloy near the martensitic transformation. J Appl Phys 51(3):1833-1834

25. Nye JF (1957) Physical properties of crystals, their representation by tensors and matrices. Clarendon Press, Oxford

26. Duerig TW (1990) Engineering aspects of shape memory alloys. Butterworth-Heinemann, London, Boston

27. Bowers M, Chen X, De Graef M, Anderson P, Mills M (2014) Characterization and modeling of defects generated in pseudoelastically deformed NiTi microcrystals. Scr Mater 78:69-72 
28. Norfleet D, Sarosi P, Manchiraju S, Wagner M-X, Uchic M, Anderson P, Mills M (2009) Transformation-induced plasticity during pseudoelastic deformation in Ni-Ti microcrystals. Acta Mater 57(12):3549-3561

29. Paranjape HM (2014) Modeling of shape memory alloys: phase transformation/plasticity interaction at the nano scale and the statistics of variation in pseudoelastic performance. The Ohio State University, Columbus

30. Pelton A (2011) Nitinol fatigue: a review of microstructures and mechanisms. J Mater Eng Perform 20(4-5):613-617

31. Pelton A, Huang G, Moine P, Sinclair R (2012) Effects of thermal cycling on microstructure and properties in Nitinol. Mater Sci Eng A 532:130-138

32. Perkins J, Sponholz RO (1984) Stress-induced martensitictransformation cycling and 2-way shape memory training in $\mathrm{Cu}-$ Zn-Al alloys. Metall Trans A 15(2):313-321

33. Chen X, Srivastava V, Dabade V, James RD (2013) Study of the cofactor conditions: conditions of supercompatibility between phases. J Mech Phys Solids 61(12):2566-2587

34. Stebner AP, Vogel SC, Noebe RD, Sisneros TA, Clausen B, Brown DW, Garg A, Brinson LC (2013) Micromechanical quantification of elastic, twinning, and slip strain partitioning exhibited by polycrystalline, monoclinic nickel-titanium during large uniaxial deformations measured via in situ neutron diffraction. J Mech Phys Solids 61(11):2302-2330

35. Benafan O, Noebe R, Padula S, Garg A, Clausen B, Vogel S, Vaidyanathan R (2013) Temperature dependent deformation of the B2 austenite phase of a NiTi shape memory alloy. Int J Plast $51: 103-121$
36. Johnson A (1979) Experimental results on a continuous-band nitinol heat engine. In: Proceedings of the nitinol heat engine conference, sponsored by US Department of Energy and US Naval Surface Weapons Center, Silver Spring

37. Benafan O, Padula SA, Noebe RD, Sisneros TA, Vaidyanathan R (2012) Role of B19 ' martensite deformation in stabilizing twoway shape memory behavior in NiTi. J App Phys 112(9):093510

38. Ii S, Yamauchi K, Maruhashi Y, Nishida M (2003) Direct evidence of correlation between $\{20-1\}\left(\mathrm{B} 19^{\prime}\right)$ and $\{114\}$ (B2) deformation twins in Ti-Ni shape memory alloy. Scr Mater 49(7):723-727

39. Nishida M, Ii S, Kitamura K, Furukawa T, Chiba A, Hara T, Hiraga K (1998) New deformation twinning mode of B19 ' martensite in Ti-Ni shape memory alloy. Scr Mater 39(12):1749-1754

40. Zhang JX, Sato M, Ishida A (2001) Structure of martensite in sputter-deposited Ti-Ni thin films containing Guinier-Preston zones. Acta Mater 49(15):3001-3010

41. Pelton AR, Schroeder V, Mitchell MR, Gong XY, Barney M, Robertson SW (2008) Fatigue and durability of Nitinol stents. J Mech Behav Biomed Mater 1(2):153-164

42. Stebner A, Bhattacharya K (2014) Micromechanics inspired, phenomenological model of fully coupled plasticity, phase transformation, and martensite reorientation in shape memory alloys. In: Bajaj A, Zavattieri P, Koslowski M, Siegmund T (eds). Proceedings of the society of engineering science 51st annual technical meeting, West Lafayette, Purdue University Libraries Scholarly Publishing Services, October 1-3, 2014. http://docs.lib.purdue.edu/ses2014/mss/cppt/3 